\title{
AN IOT BASED APPROACH IN PARALYSIS PATIENT HEALTHCARE SYSTEM
}

\author{
Prajakta A. Jadhav, Snehal B. Jadhav, Amruta P. Erudkar, Sayali A. Bhurke, Nayana S. Palekar \\ (UG students, Department of Computer Science and Engineering)
}

\author{
Prof. M. A. Pardesi, Project Guide
}

\begin{abstract}
In today's world many people are suffering from physical disabilities due to paralysis or some accidental problem. Most of these patients are dependent on care taker. Paralysis is a condition in which there is impairment of one or more muscles in body. In order to assist these patients' microcontrollerbased circuitry plays major role in system. Sometime appliances are also controlled by patient's hand motion. This system also monitors patient's heartbeat, if it exceeds normal value then buzzer will be activated and message will be shown on LCD to doctors and caretakers to attend patient.
\end{abstract}

Keywords: IOT, embedded system, cloud, healthcare automation, home automation, web application.

\section{INTRODUCTION}

Paralytic patient healthcare system is a system designed to help patient convey various messages to other people like doctor, nurse or family member or caretaker, the system makes use of micro controller based circulate to achieve this functionality.

Physically disabled people many of the times rely on others, even to perform simple action like switching on/off lights, turning on/off fan etc. In order to provide solutions to these activities the system use hand motioncontrolled device when even there is motion the rely circuit will be activated which time on/off lights and fan.

Patient healthcare system is method in which the doctor or care taker will monitor patient's health from any location any time. There are cases when there is no one hereby him/her to overcome such situation is system continuously monitors health records of patients such as heartbeat and body temperature.

\section{PROPOSED METHODOLOGY}

The proposed system is divided into three parts, Patient Data Acquisition \& Device Control (Input, Output \& Processing System), Doctor's Panel, Patient Panel (SelfMonitoring \& Device Control). Patient data acquisition and device control, Whole hardware setup forms first module. This is the main \& primary module of presented system. It collects the sensor's data and feeds to the IoT enabled microcontroller which acts as a brain of this system. This brain sends all data to web application where doctor \& patient can login to monitor health. Microcontroller has Wi-Fi chipset to perform the connectivity with webserver via Wi-Fi network present at patient's place. This module also performs the task of appliance control in patient's room on his requests being generated from web application itself. Doctor's Panel, this is the part of web application where only doctor can login to view all the data of all his/her patients. This data includes the threshold values of patient's health parameters being sensed by sensors, alert limits, reminders etc. All the critical data records of patient will be stored here $\&$ are accessible only by doctor.

Patient Panel, this is the part of web application where only patient can login to monitor his routine health check records and the threshold values as well as reminders set by his doctor. This panel also gives functionality to patient to control devices in his room which are connected to this system 


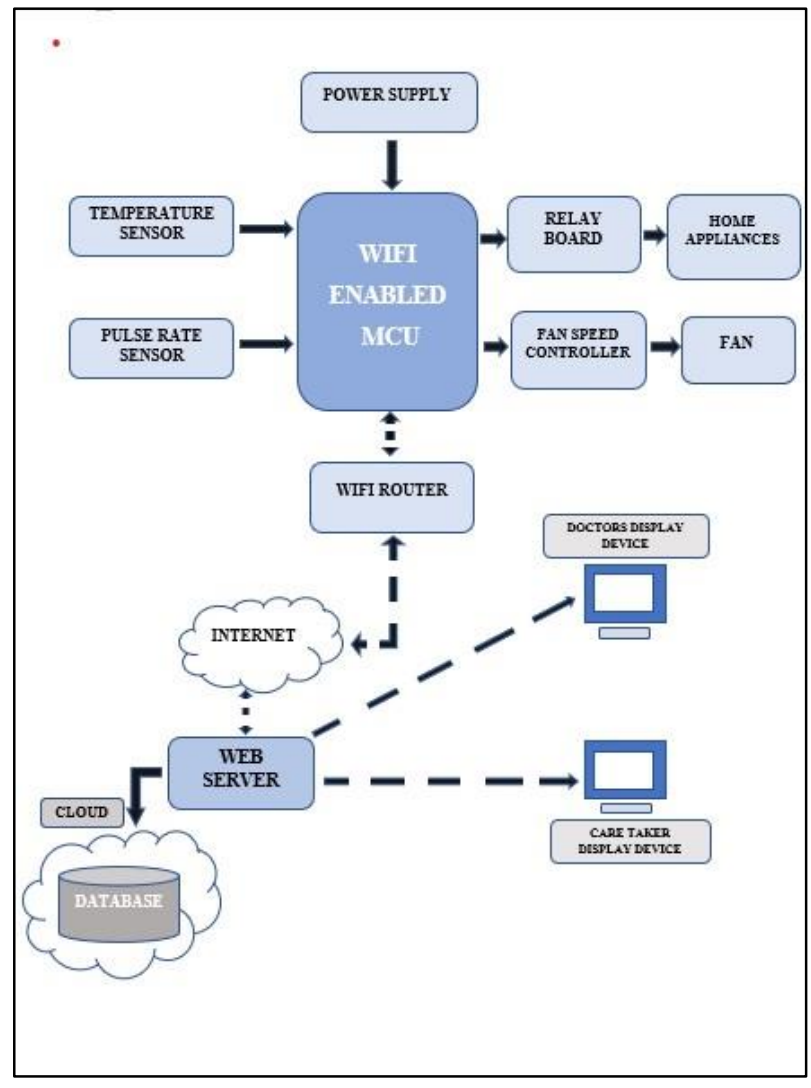

Fig 1: Block Diagram

\section{HARDWARE IMPLEMETATION}

In this part we will be briefly explain about the components and their specifications which we have used to make the system. It consists of two sensors, one relay board, esp32 microcontroller, battery of 5-volt, small fan and bulb (for demonstration purpose) and other necessary electrical device to make connections.

\section{Sensors:}

\section{DS18B20 Temperature Sensor:}

The DS18B20 is one type of temperature sensor and it supplies 9-bit to 12-bit readings of temperature. These values show the temperature of a particular device. The communication of this sensor can be done through a onewire bus protocol which uses one data line to communicate with an inner microprocessor. Additionally, this sensor gets the power supply directly from the data line so that the need for an external power supply can be eliminated. The applications of the DS18B20 temperature sensor include industrial systems, consumer products, systems which are sensitive thermally, thermostatic controls, and thermometers.

\section{Pulse sensor:}

When a heartbeat occurs, blood is pumped through the human body and gets squeezed into the capillary tissues. The volume of these capillary tissues increases as a result of the heartbeat. But in between the heartbeats (the time between two consecutive heartbeats,) this volume inside capillary tissues decreases. This change in volume between the heartbeats affects the amount of light that will transmit through these tissues. This change is very small but we can measure it with the help of Arduino.

The pulse sensor module has a light which helps in measuring the pulse rate. When we place the finger on the pulse sensor, the light reflected will change based on the volume of blood inside the capillary blood vessels. During a heartbeat, the volume inside the capillary blood vessels will be high. This affects the reflection of light and the light reflected at the time of a heartbeat will be less compared to that of the time during which there is no heartbeat (during the period of time when there is no heartbeat or the time period in between heartbeats, the volume inside the capillary vessels will be lesser. This will lead higher reflection of light). This variation in light transmission and reflection can be obtained as a pulse from the output of pulse sensor. This pulse can be then conditioned to measure heartbeat and then programmed accordingly to read as heartbeat count. 


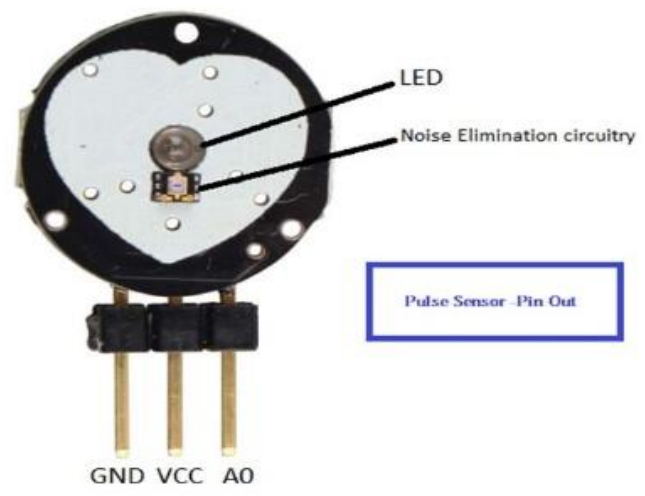

\section{Relay boards:}

Relay boards are computer boards with an array of relays and switches. They have input and output terminals and are designed to control the voltage supply. Relay boards provide independently programmable, real-time control for each of several onboard relay channels.

Relay boards are used in many different applications. Some products are used to control lights, motors, and other electronic devices in industrial and commercial applications. Others are also used to control heater temperatures, or are used in power switching applications Relay boards that are designed for specific types of industrial equipment are also available.

\section{ESP32 Development board:}

ESP32 Development board is based on the ESP WROOM32 WIFI + BLE Module. It's a low-footprint, minimal system development board powered by the latest ESP-WROOM-32 module and can be easily inserted into a solderless breadboard. It contains the entire basic support

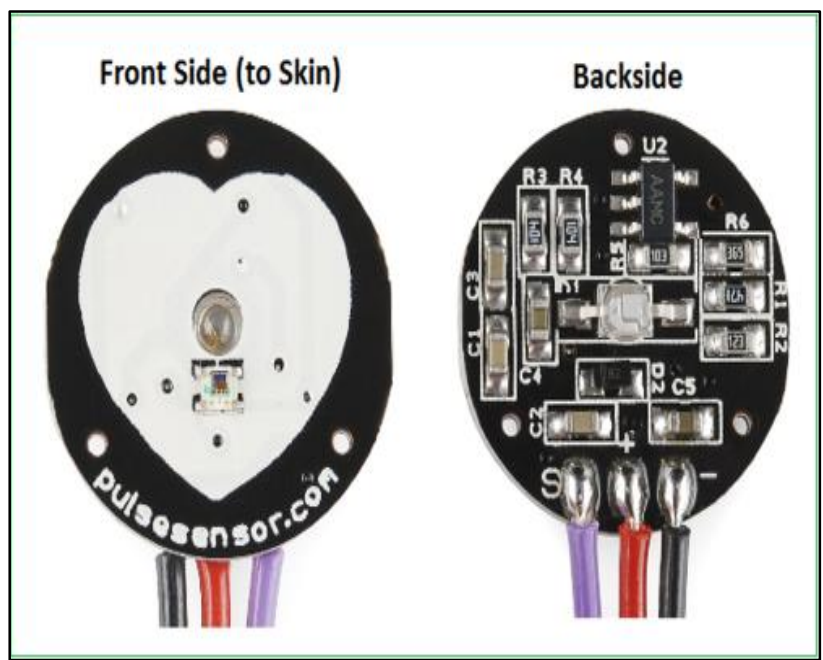

circuitry for the ESP-WROOM-32, including the USBUART bridge, reset- and boot-mode buttons, LDO regulator and a micro-USB connector. Every important GPIO is available to the developer.

\section{Motor Driver:}

L298N 2A Based Motor Driver is a high-power motor driver perfect for driving DC Motors and Stepper Motors. It uses the popular L298 motor driver IC and has an onboard $5 \mathrm{~V}$ regulator which it can supply to an external circuit. It can control up to 4 DC motors, or 2 DC motors with directional and speed control.

\section{Features:}

Driver chip: L298 dual H-bridge driver chip. Operates up to $35 \mathrm{~V}$ DC Drive part of the peak current Io: 2A / Bridge Logical part of the terminal power supply range Vss $: 4.5 \mathrm{~V}-5.5 \mathrm{~V}$

Logical part of the operating current range: $0 \sim 36 \mathrm{~mA}$ Maximum power consumption: $20 \mathrm{~W}$. 


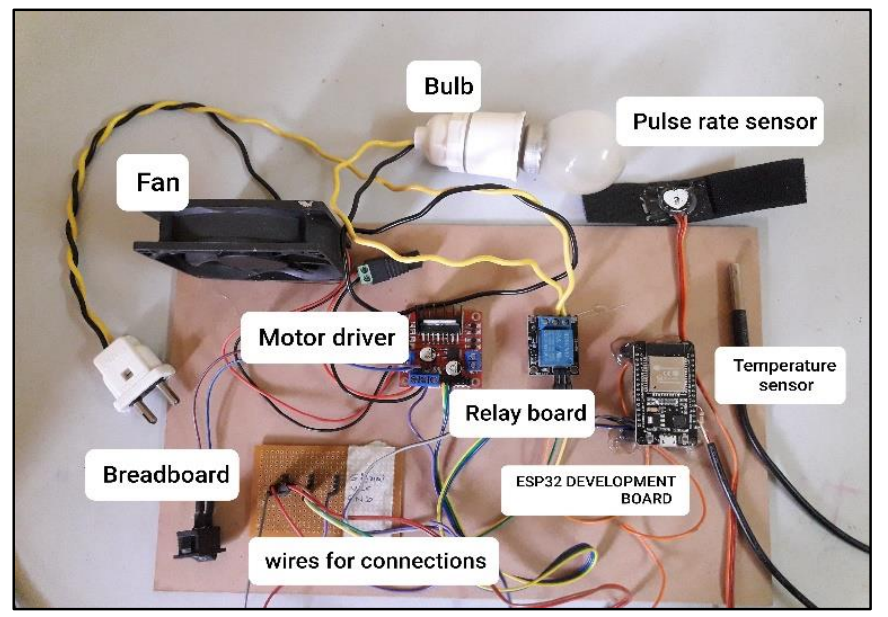

Fig 2: Microcontroller based circuitry

\section{IV.}

\section{SOFTWARE IMPLEMETATION}

In this part we will be explaining about the software's required to completed the whole project. We have used ARDUINO IDE (Integrated Developed Environment). The Arduino Integrated Development Environment (IDE) is a cross-platform application (for Windows, macOS, Linux) that is written in functions from $\mathrm{C}$ and $\mathrm{C}++$. It is used to write and upload programs to Arduino compatible boards, but also, with the help of third-party cores, other vendor development boards. The language which used for coding is "embedded c".

The second software named "FileZilla" is used for file transferring. FileZilla Client is a free and open-source FTP, file transfer protocol, tool that functions with FTPS and SFTP. The intuitive user interface allows for people to easily transfer and download files with their devices and hosting accounts within the terms of the GPL: General Public License. For a free FTP and FTPS server, FileZilla offers their server as an open-source service.
The third software we used is" HeidiSQL". HeidiSQL is a useful and reliable tool designed for web developers using the popular MySQL server, Microsoft SQL databases and PostgreSQL. It enables you to browse and edit data, create and edit tables, views, procedures, triggers and scheduled events. Also, you can export structure and data either to SQL file, clipboard or to other servers.

\section{Screenshots of the websites:}

\section{Admin Login:}

The person who will be appointed in hospital to operate the whole system will be given access to admin module. $\mathrm{He} / \mathrm{she}$ will be able to update the information such as new doctors who will be appointed in future, to enrol new patient name in the system, to add newly devices which are required in hospital for medication, what are departments and the names of staff appointed for various departments.

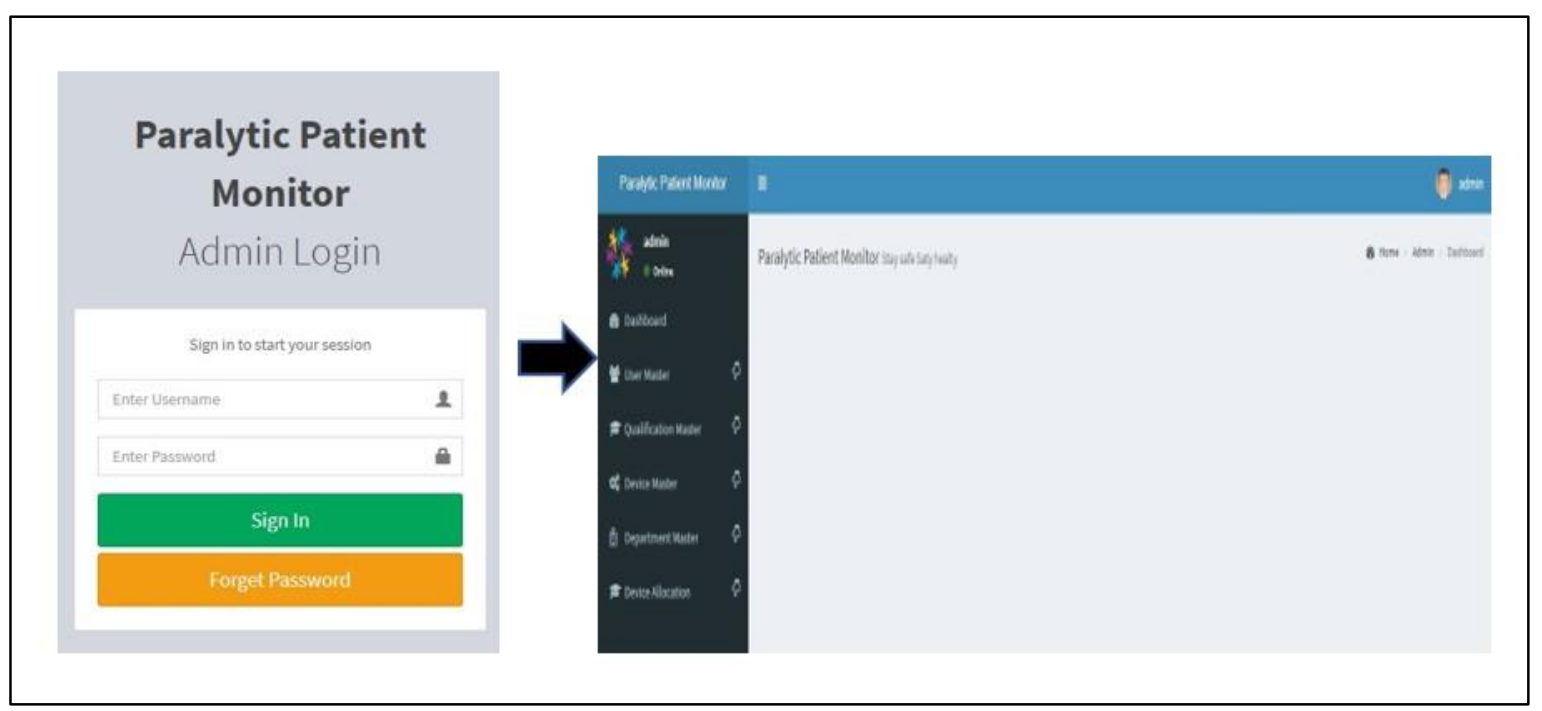

Fig 3: Admin Login 


\section{Patient Login:}

In patient login the caretaker will be able to access once the patient get enrolled in the system. He/she will be provided with the login credentials, so that they can see patients value from anywhere they want. Here data from sensor will be displayed on the screen to care taker and also to the doctors, so that they can monitor the patient's health by sitting in cabin, or if the patient is in home, then also this system will provide data of patients on website.

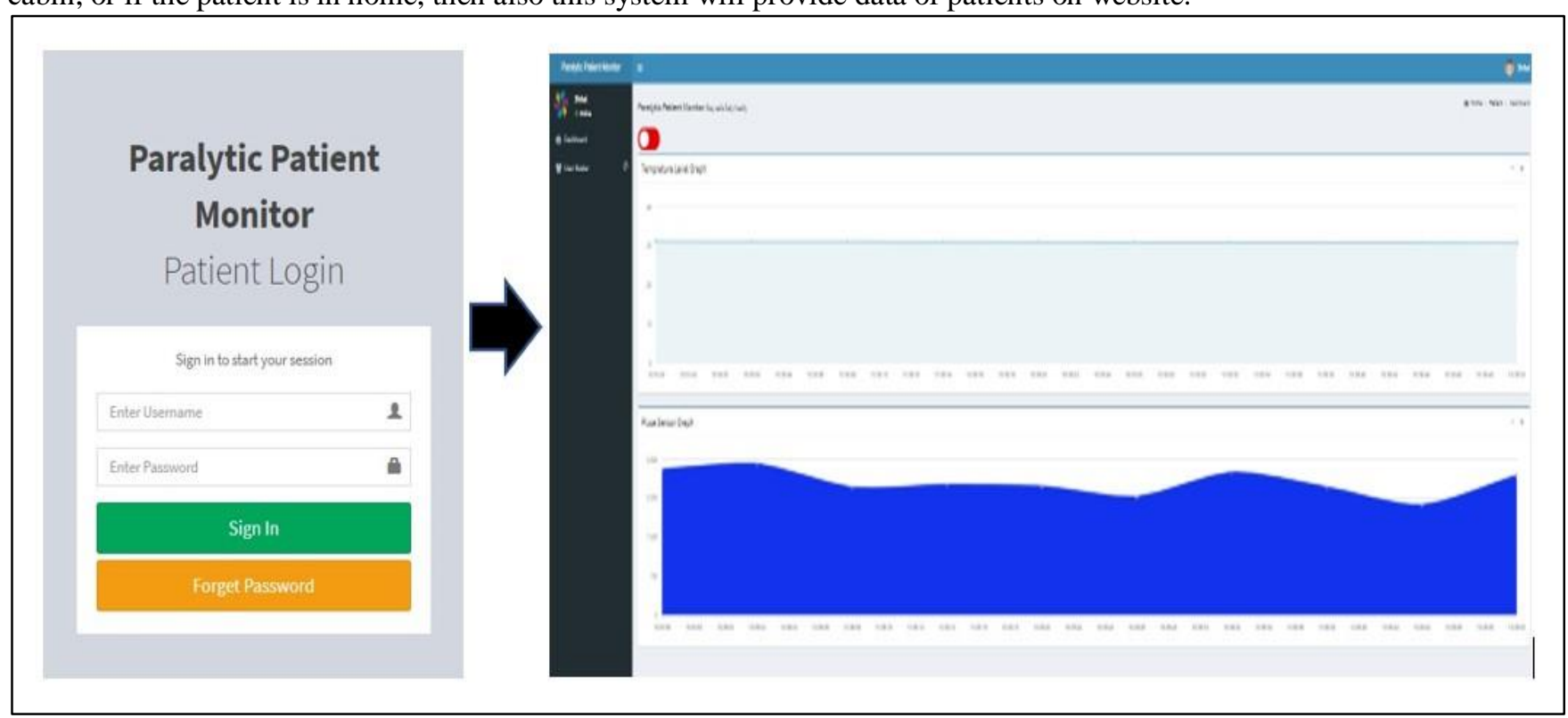

Fig 4: Patient Login

\section{Doctor's Login:}

Here the access will be only given to the doctor to monitor the patient's health. Patient's name will be in the list format so to check particular patient's health. If doctor will see the patient health values are exceeding the limit, then he will quickly notify the caretaker and go at the patient's place. From here he can also check what are the devices allocated for different patient.

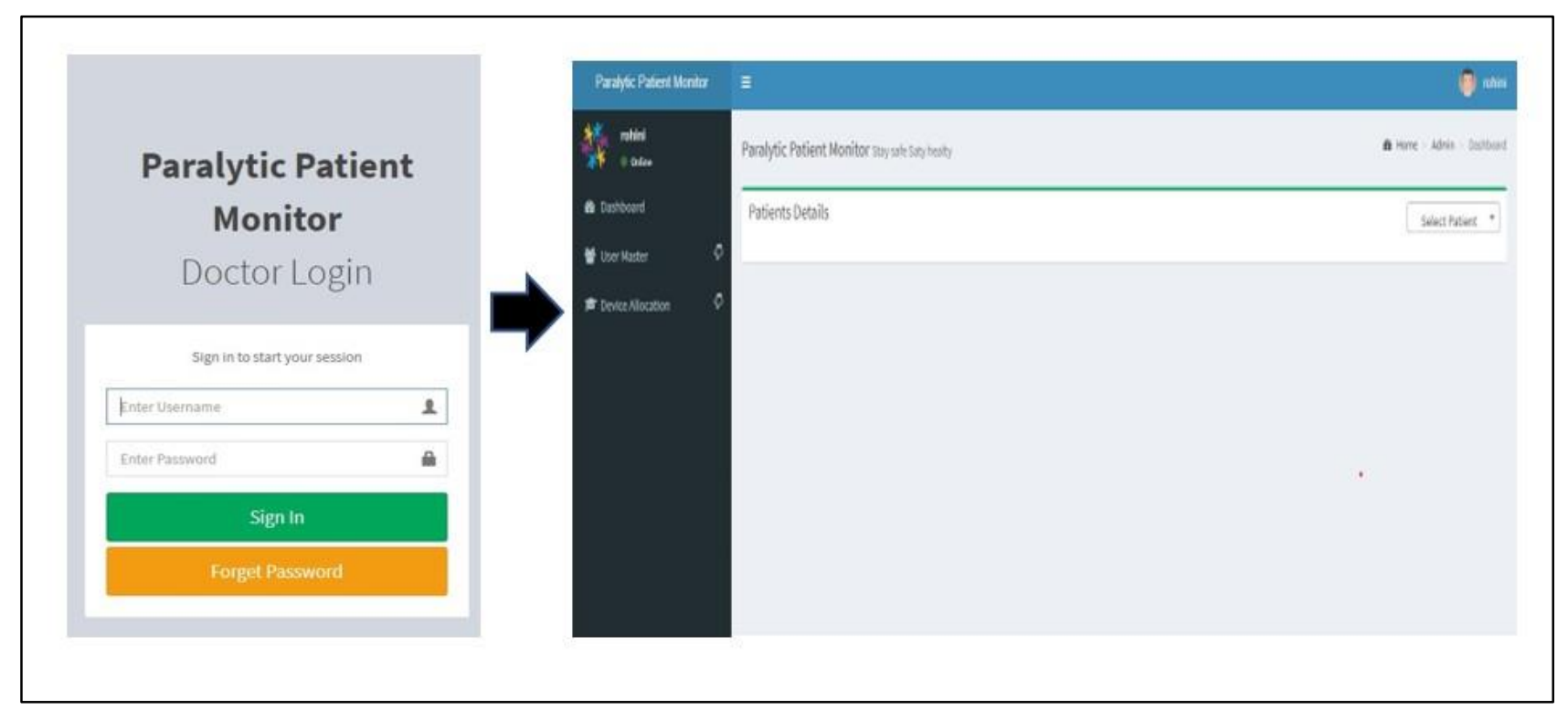

Fig 5: Doctor's Login 


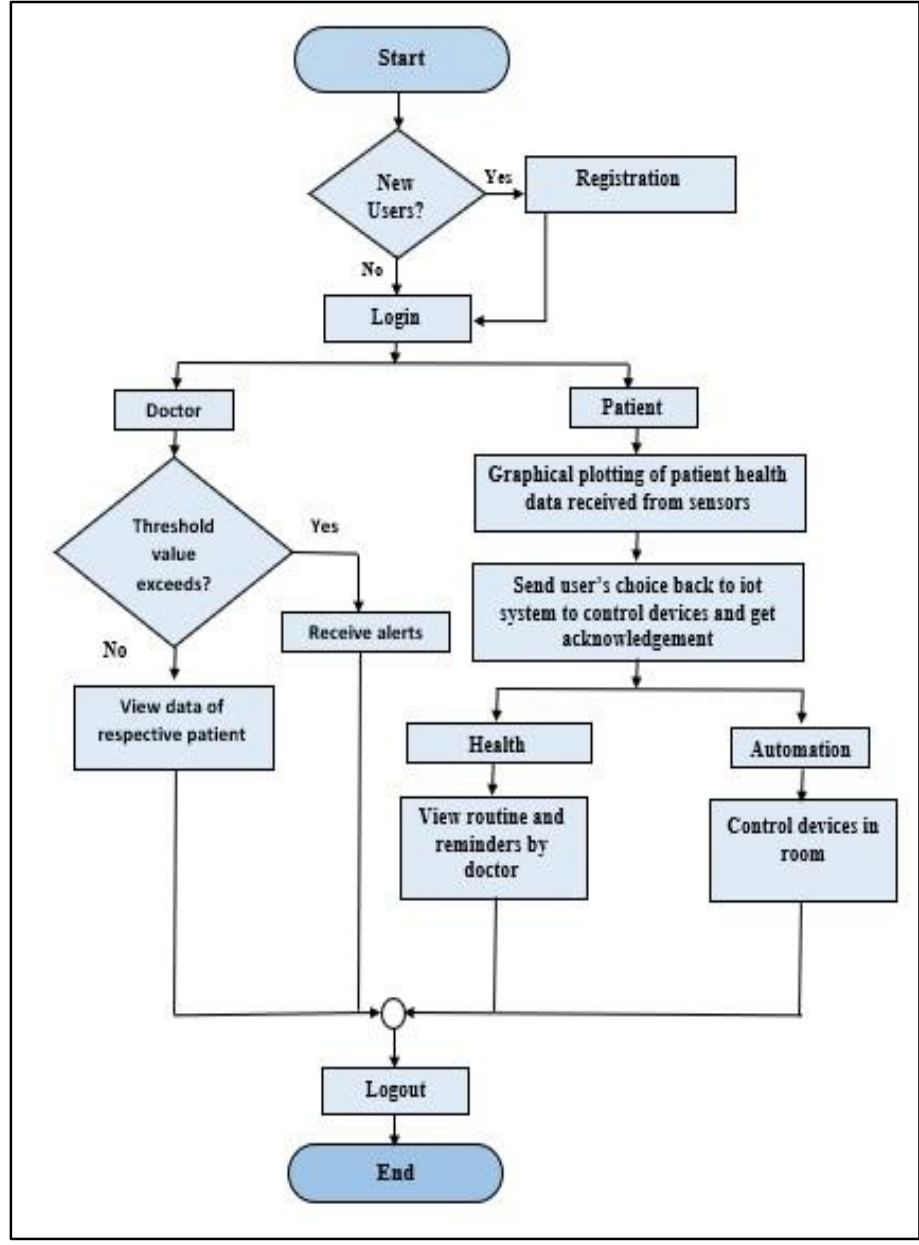

Fig 6: Flowchart

\section{WORKING \& RESULTS:}

The proposed system is not only helpful for the paralytic patients but also for old age peoples and temporarily disabled people. The results of temperature sensor and pulse sensor are shown figure,

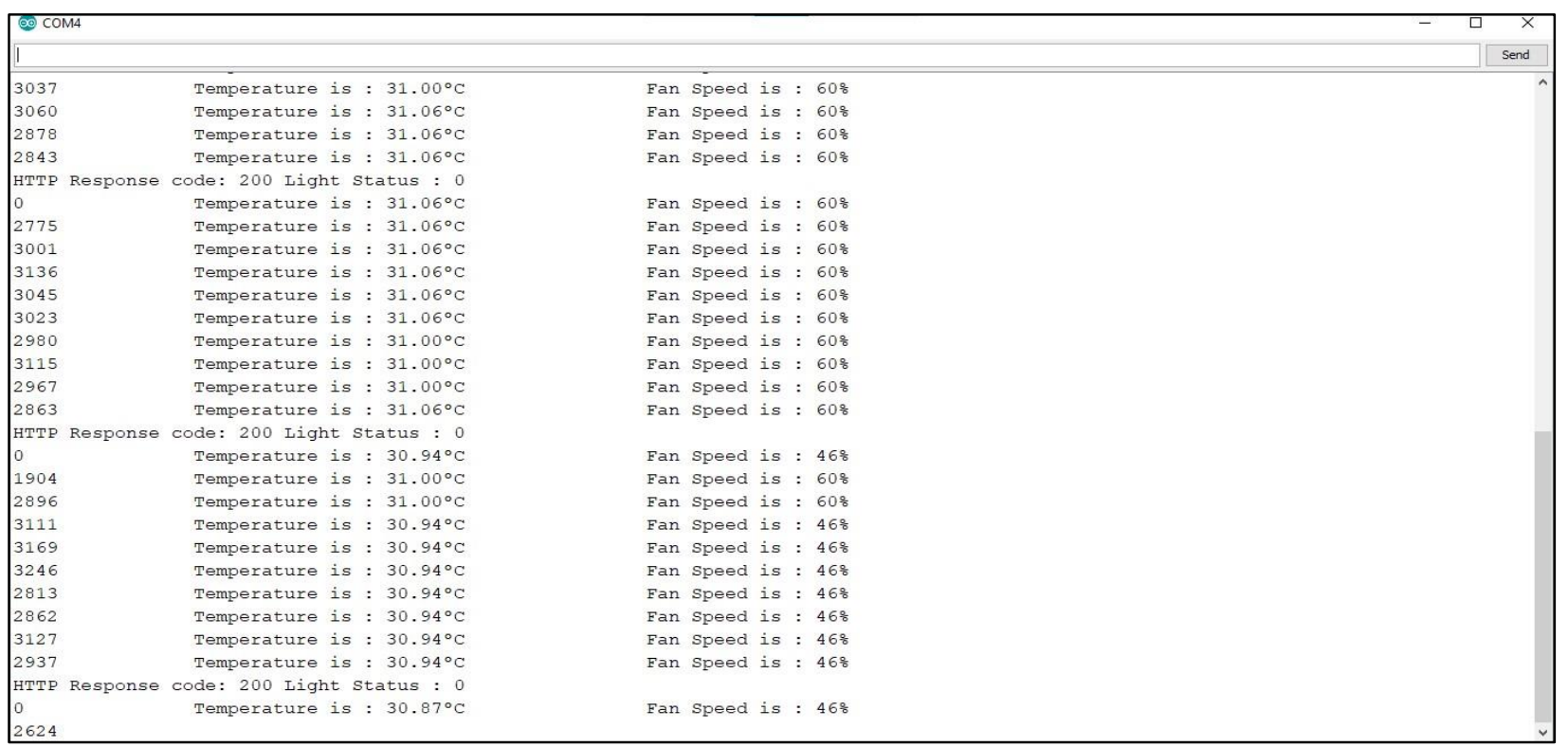

Fig 7: Reading of temperature sensor over serial monitor and change in fan speed depending on temperature sensor value. 


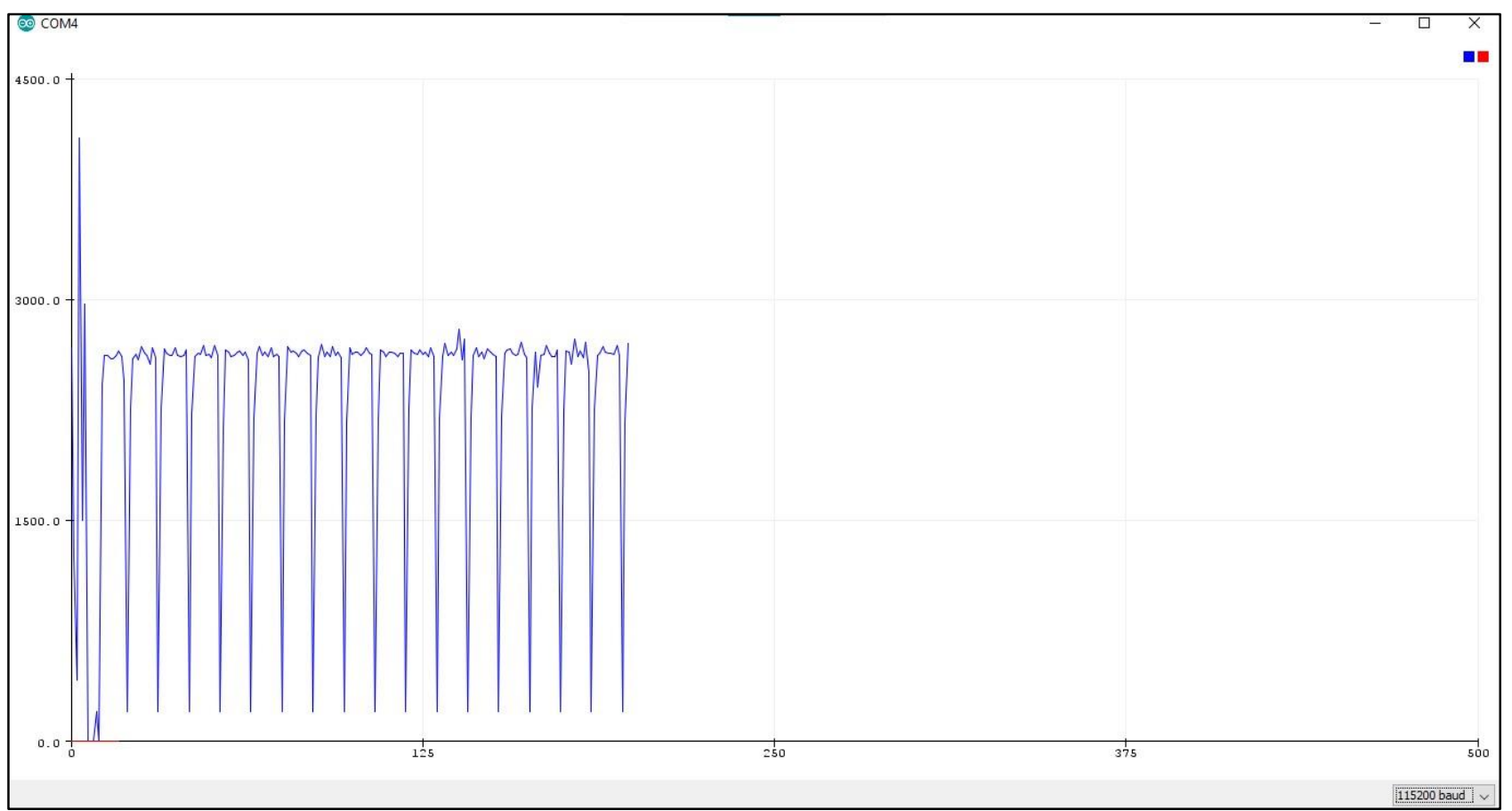

Fig 8: Readings of pulse rate sensor over serial plotter.

\section{Working of the proposed system:}

1. Step 1: We have to install Arduino ide for coding purpose. Then perform the coding part according to your components used and required output. Once the coding part is done, we have to compile and upload it, then we will get to see the outputs on serial monitor or on serial plotter.

2. Step 2: Once the circuitry is completed ok then we have to go for web part.

3. Step 3: In web part there are 3 modules doctor module, patient module and admin module as discuss above. For accessing a particular system, he/she has to be enrolled in the system as admin, as patient (or caretaker) or as doctor.

4. Step 4: Once all the setup is done, we have to start the server (currently we are storing database locally).

5. Step 5: After all the above processes are done successfully, then the sensor data will be passed to website.

6. Step 6: Data coming from sensors which are mounted on patient body will be given to the system and the doctor will be able to monitor patient's health. If there are some extreme situations then alerts will be given to patient as well as doctor and can take actions accordingly.

\section{CONCLUSION AND FUTURE SCOPE:}

The proposed system will be helping hand for paralytic patient, old age and temporarily disabled people. The patients can convey his/her needs through the designed system. Home automation is also included in proposed system so that patient can control different home appliances without help of care taker. Health monitoring is the major issues, this system will continuously monitor patients body temperature and pulse rate. If the temperature or pulse rate exceeds normal value then the messages will be quickly notified to the care taker and doctor. In future, considering the COVID-19 pandemic more health parameters can be added.

\section{REFERENCES}

1. Akhil Reji George, Harold Gerard, Sojy Rajan, Gowri Gopal, Radhika S Krishnan.” Smart Amigo-An Assistant for Paralytics" published in International Journal of Engineering Research \& Technology (IJERT) Thiruvananthapuram, India Volume: 9 Issue 07, July-2020.

2. S. A. C. Aziz, A. F. Kadmin, N. Rahim, W. H. W. Hassan, I. F. A. Aziz, M. S. Hamid, R. A. Hamzah." Development of automatic healthcare instruction system via movement gesture sensor for paralysis patient" published in International Journal of Electrical and Computer Engineering (IJECE) Vol. 9, No. 3, June 2019.

3. Akansha Singh, Deepa Gupta, Neetu Mittal. "Enhancing Home security systems Using IOT" International Conference on Electronics Communication and Aerospace Technology [ICECA 2019] Volume: 05, Issue: 95, Feb- 2019.

4. Kumara K R, Ankita Kadam, Neha Rane, Shraddha Vernekar, Asma Gouda.” Sensor Based 
International Journal of Engineering Applied Sciences and Technology, 2021

Vol. 6, Issue 3, ISSN No. 2455-2143, Pages 238-245

Published Online July 2021 in IJEAST (http://www.ijeast.com)

Wearable System to Assist Paralytic Patient with

Continuous Health Monitoring" published in International Journal on Future Revolution in
Computer Science \& Communication Engineering, Karnataka, INDIA. Volume: 4 Issue 